\title{
Expression of CDX2 and Hepatocyte Antigen in Benign and Malignant Lesions of Gallbladder and Its Correlation with Histopathologic Type and Clinical Outcome
}

\author{
Qing-Long Li • Zhu-Lin Yang • Jie-Qiong Liu • \\ Xiong-Ying Miao
}

Received: 27 May 2010 / Accepted: 1 December 2010 / Published online: 8 January 2011

(C) The Author(s) 2011. This article is published with open access at Springerlink.com

\begin{abstract}
Recent studies have shown that both CDX2 and Hepatocyte antigen (Hep) are detected in different types of cancer and associated with clinical prognosis. However, fever studies have examined gallbladder cancer specimens, and little is known about the clinicopathological significance of both CDX2 and Hep expression in gallbladder adenocarcinomas. In present study, we examined the expression frequencies of CDX2 and Hepatocyte antigen (Hep), and explored their clinicopathologic significances in gallbladder adenocarcinoma. Immunohistochemistry was used to detect and compare the frequencies of CDX2 and Hep expression in 108 samples of gallbladder adenocarcinoma, 46 peri-tumor tissues and 35 chronic cholecystitis. The expression frequencies for CDX2 and Hep were 49/ 108 (45.4\%) and 45/108 (41.7\%) in gallbladder carcinoma; $13 / 46(28.3 \%)$ and $11 / 46(23.9)$ in peri-tumor tissues; $5 / 35$ $(14.3 \%)$ and $2 / 35(5.7 \%)$ in chronic cholecystitis. The positive staining of CDX2 or Hep in gallbladder adenocarcinoma was significantly higher than that in peritumoral tissues (both, $P<0.05$ ), and chronic cholecystits (both, $P<$ 0.01). The expression of CDX2 or Hep was negatively correlated to grade of differentiation, tumor size and lymph node metastasis $(P<0.01$ or $P<0.05)$. Elevated expression frequency of CDX2 or Hep was associated with increased overall survival $(P=0.003$ or $P=0.002)$. Multivariate $\mathrm{Cox}$ regression analysis showed that $\mathrm{CDX} 2(P=0.014)$ or Hep $(P=0.026)$ expression was an independent prognostic
\end{abstract}

\footnotetext{
Q.-L. Li · Z.-L. Yang $(\bowtie) \cdot J .-Q$. Liu $\cdot$ X.-Y. Miao

Research Laboratory of Hepatobiliary Diseases,

The Second Xiangya Hospital of Central South University,

139 Middle Renmin Road,

Changsha, Hunan 410011, China

e-mail: zhulin.yang@yahoo.com
}

predictor in gallbladder adenocarcinoma. CDX2 and Hep might function as important biological markers in the development and prognosis of gallbladder adenocarcinoma.

Keywords Gallbladder cancer - Chronic cholecystitis . CDX2 $\cdot$ Hepatocyte antigen (Hep) $\cdot$ Hepatocyte paraffin 1 . Immunohistochemistry $\cdot$ Clinicopathology
Abbreviations
CDX2 Caudal-related homeobox transcription factor Hep Hepatocyte antigen
Hep Par 1 Hepatocyte paraffin 1 monoclonal antibody

\section{Introduction}

Gallbladder cancer is often a terminal malignancy occurring predominantly in the elderly women. It accounts for nearly two-thirds of the biliary tract cancers, making it the most common primary biliary cancer and the fifth most common cancer of the gastrointestineal tract $[1,2]$. More than $85 \%$ of gallbladder cancers belong to adenocarcinomas often well or moderately differentiated, and the rest approximately $15 \%$ are squamous, adenosquamous or undifferentiated carcinoma. To date surgery is the only potentially curative modality for patients with gallbladder cancer, however, more than $70 \%$ of cases is unresectable owing to local invasion and metastasis. A better understanding of pathogenesis and clinicopathological charateristics of gallbladder cancer may provide insights for early diagnosis and treatment improvement for this deadly disease.

Caudal type homeobox transcription factor CDX2 is mostly expressed in small and large intestinal epithelial 
cells and plays an essential role in regulating proliferation and differentiation of the intestinal epithelium [3, 4]. CDX2 expression has been considered as a sensitive marker of intestinal metaplasia in the esophagus $[5,6]$ and stomach $[7,8]$. Recent studies have shown that CDX2 expression is also detected in intestinal-type gastric carcinoma, esophageal carcinoma, and other malignancies such as cholangiocarcinoma and intraductal papillary neoplasia of the liver [9-14]. In gallbladder, CDX2 expression is not seen in the normal tissue, however it has been detected in the intestinal metaplasia mucosa in cholelithiasis [15], and also in adenocarcinoma [16, 17]. Few studies reported the relationship between CDX2 expression and prognosis of gallbladder adenocarcinoma [16]. The clinical and pathological significance of CDX expression in gallbladder adenocarcinoma remains to be further elucidated.

Hepatocyte antigen (Hep) is detected by Hep Par 1, a monoclonal antibody developed from Hepatic tissue from a failed live allograft and considered to be specific for normal and neoplastic Hepatocytes. Therefore, Hep has emerged as a diagnostic marker in surgical histopathology to distinguish Hepatocellular carcinoma (HCC) from liver metastases of other carcinomas $[18,19]$. However subsequent studies has revealed that Hep expression has been detected in cholangiocarcinomas $[20,21]$, gallbladder carcinomas [22, 23], gastric carcinomas and others [21-25]. To our knowledge, the clinicopathological significance of Hepatocyte antigen expression in gallbladder adenocarcinoma has not been reported.

In the present study, we examined the expression frequency of CDX2 and Hepatocyte antigen in 108 specimens of gallbladder adenocarcinoma, 46 of peri-tumor tissues and 35 of chronic cholecystitis by means of immunohistochemistry with attempt to better understand the reasons for CDX2 and Hep expression in lesions of gallbladder and its clinicopathological implications.

\section{Materials and Methods}

\section{Specimens and Clinicopathologic Materials}

Gallbladder carcinoma resection specimens were collected from 108 cases hospitalized in the Second and First Xiangya Hospital of Central South University, and People's Hospital of Hunan Province (Changsha, China) from June 1996 through June 2006. Of the 108 cases with gallbladder carcinoma, 31 was male $(28.7 \%)$ and 77 was female $(71.3 \%)$. Ages of patients were from 35 to 70 years old with an average $52.6 \pm 11.2$. The pathological types of these cases were all adenocarcinoma, including 9 cases $(8.2 \%)$ of adenomatous canceration, 29 cases $(26.9 \%)$ of welldifferentiated adenocarcinoma, 29 cases $(26.9 \%)$ of moderately-differentiated adenocarcinoma, 30 cases
(27.8\%) of poorly-differentiated adenocarcinoma, and 11 cases $(10.2 \%)$ of mucinous adenocarcinomas. There were 59 cases $(54.6 \%)$ with peri-gallbladder infiltrations, 59 $(54.6 \%)$ with regional lymph node metastasis, and 58 $(57.3 \%)$ with gallbladder cancer complicated with gallstones. There were 34 cases $(31.5 \%)$ with radical resection, $48(44.4 \%)$ with palliative operation, and $26(24.1 \%)$ with no operation because of distant metastasis. In 46 selected peritumoral tissues (away from tumor $\geq 3 \mathrm{~mm}$ ) from some of above patients, 10 were normal, 10 were mild atypical hyperplasia, 12 were middle atypical hyperplasia, and 14 were severe atypical hyperplasia. And another 35 cases of chronic cholecystitis specimens (15 cases of simple chronic cholecystitis and 20 cases of chronic cholecystitis complicated with gallstones) were also selected as control, in which there were 11 cases of normal tissues, 12 cases of mild atypical hyperplasia, 7 cases of middle atypical hyperplasia and 5 cases of severe atypical hyperplasia. All these specimens were fixed by $4 \%$ formaldehyde, followed by conventional paraffin-embedded sectioning.

\section{Immunohistochemical Staining}

Mouse anti-human CDX2 and Hep Par 1 monoclonal antibodies and EnVision ${ }^{\mathrm{TM}}$ Detection Kit were from Dako Laboratories, California, USA. The staining of CDX2 and Hep were carried out according to the manufacture's protocol (ChemMate ${ }^{\mathrm{TM}}$ EnVison +/HRP/DAB, Rabbit/ Mouse Two Step Staining Method). Briefly, the sections were deparaffined and incubated with peroxidase inhibitor $\left(3 \% \mathrm{H}_{2} \mathrm{O}_{2}\right)$ in dark for $15 \mathrm{~min}$, followed by EDTAtrypsinase digestion liquid $(0.125 \%, \mathrm{pH} 9.0)$ for $15 \mathrm{~min}$, then soaked with PBS liquid (pH7.4) three times with each for $5 \mathrm{~min}$. The pretreated sections were then incubated with mouse anti-CDX2 or -Hep Par 1 for 60 min under room temperature. After three rinses with PBS, solution A was added to the sections, and incubated for $30 \mathrm{~min}$ at room temperature. Substrate DAB was added and followed by hematoxylin counter-staining. The Slides were dehydrated with different concentrations $(70 \%-100 \%)$ of alcohol, and soaked in xylene for three times with each $5 \mathrm{~min}$. and finally mounted with neutral balsam. The readouts of CDX2 or Hep staining were considered as positive when more than $20 \%$ of the tumor cells were stained, otherwise as negative. The positive controls were the positive sections that provided by Dako, and the negative controls were the $0.01 \mathrm{~mol} / \mathrm{L}$ PBS liquid ( $\mathrm{pH} 7.4$ ) (substitute the first antibody).

\section{Statistically Analysis}

The SPSS13.0 program was used for calculation of interrelationships between the analyzed CDX2 or Hep and 

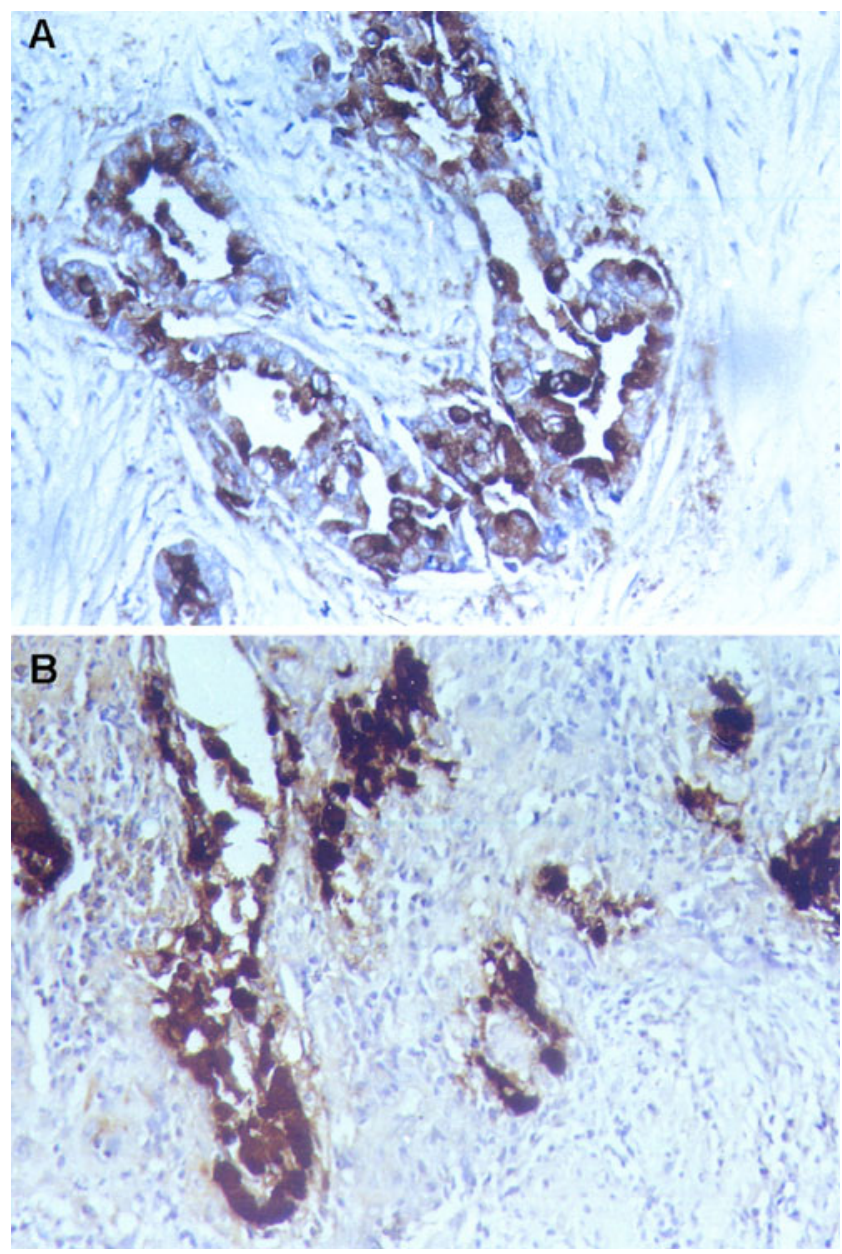

Fig. 1 Immunohistochemical staining of CDX2 in gallbladder adenocarcinoma and peritumoral tissue $(\times 200)$. a CDX2 positive staining was observed in nuclei of a representative moderatelydifferentiated gallbladder adenocarcinoma. b CDX2 expression was located in nuclei of a representative peritumoral tissue with severelyatypical hyperplasia

histological or clinical factors by $\chi 2$ independence test. Fisher's exact probability test was also used for analyzing statistical association between the two independent sample groups. The results were considered to be significant when the $P$ value were less than 0.05 . Disease specific overall survival analyses were determined and compared using the Kaplan-Meier method and the log-rank test. For multivariate analysis the Cox regression method was performed. $95 \%$ confidence intervals were used overall.

\section{Results}

Expressions of CDX2 and Hep in Benign and Malignant Lesions of Gallbladder

We examined expression of CDX2 and Hep by immunostaining in 108 cases of gallbladder adenocarcinoma, 46
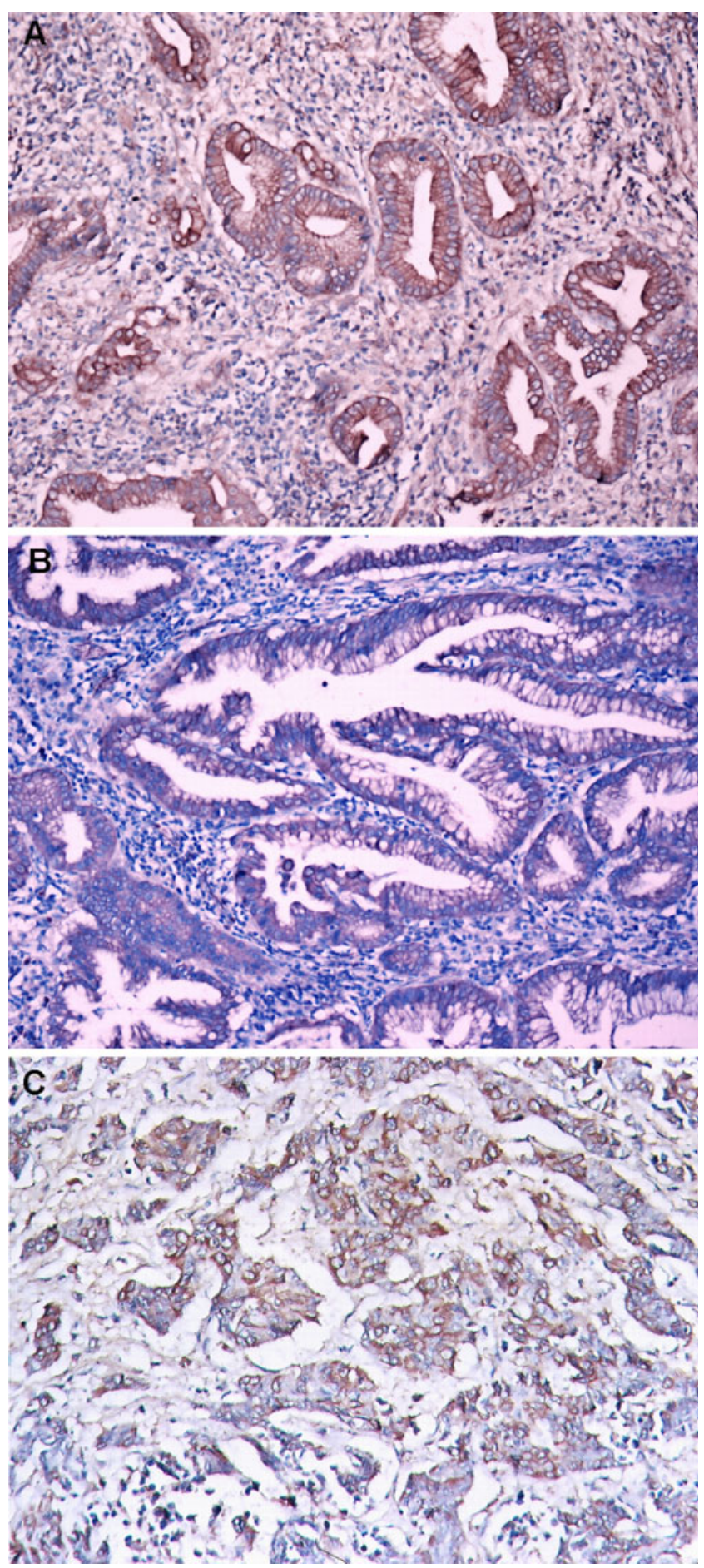

Fig. 2 Expression of Hep in gallbladder adenocarcinoma and chronic cholecystitis $(\times 200)$. a Positive staining of Hep was seen in cytoplasms in a representative well-differentiated adenocarcinoma. b Positive expression of Hep was detected in cytoplasms of a representative chronic cholecystitis with moderately-atypical hyperplasia. c Typical Hep staining of hepatocytes in a liver section 
Table 1 Expressions of CDX2 and Hep and their correlation with clinicopathologic parameters of gallbladder adenocarcinoma

\begin{tabular}{|c|c|c|c|c|c|}
\hline Group & $\mathrm{n}$ & $\mathrm{CDX} 2+(\%)$ & $P$-value $\left(\chi^{2}\right.$-test $)$ & Hep Par $1+(\%)$ & $P$-value $\left(\chi^{2}\right.$-test $)$ \\
\hline \multicolumn{6}{|l|}{ Sex } \\
\hline Male & 31 & $14(45.2)$ & \multirow[t]{2}{*}{$>0.05$} & $14(45.2)$ & \multirow[t]{2}{*}{$>0.05$} \\
\hline Female & 77 & $35(45.5)$ & & $31(40.3)$ & \\
\hline \multicolumn{6}{|l|}{ Age } \\
\hline$\leq 45$ & 24 & $11(45.8)$ & \multirow[t]{2}{*}{$>0.05$} & $10(41.7)$ & \multirow[t]{2}{*}{$>0.05$} \\
\hline$>45$ & 84 & $38(45.2)$ & & $35(41.7)$ & \\
\hline \multicolumn{6}{|l|}{ Pathologic type } \\
\hline Adenomatous canceration & 9 & $8(88.9)$ & \multirow[t]{5}{*}{$<0.01$} & $7(77.8)$ & \multirow[t]{5}{*}{$<0.05$} \\
\hline Well-differentiated & 29 & $16(55.2)$ & & $16(55.2)$ & \\
\hline Moderately-differentiated & 29 & $13(44.8)^{\mathrm{a}}$ & & $12(41.4)^{\mathrm{a}}$ & \\
\hline Poorly-differentiated & 30 & $7(23.3)^{\mathrm{b}, \mathrm{c}}$ & & $6(20.0)^{b, c}$ & \\
\hline Mucinous adenocarcinoma & 11 & $5(45.5)$ & & $4(36.4)$ & \\
\hline \multicolumn{6}{|l|}{ Tumor diameter } \\
\hline$<2.0 \mathrm{~cm}$ & 31 & $21(67.7)$ & \multirow[t]{2}{*}{$<0.01$} & $18(58.1)$ & \multirow[t]{2}{*}{$<0.05$} \\
\hline$\geq 2.0 \mathrm{~cm}$ & 77 & $28(36.4)$ & & $27(35.1)$ & \\
\hline \multicolumn{6}{|l|}{ Lymph node metastasis } \\
\hline No & 49 & $31(63.2)$ & \multirow[t]{2}{*}{$<0.01$} & $26(53.1)$ & \multirow[t]{2}{*}{$<0.05$} \\
\hline Yes & 59 & $18(30.1)$ & & $19(32.2)$ & \\
\hline \multicolumn{6}{|l|}{ Surrounding tissue invasion } \\
\hline No & 49 & $30(61.2)$ & \multirow[t]{2}{*}{$<0.01$} & $24(49.0)$ & \multirow[t]{2}{*}{$>0.05$} \\
\hline Yes & 59 & $19(32.2)$ & & $21(35.6)$ & \\
\hline \multicolumn{6}{|l|}{ Gallstones } \\
\hline No & 50 & $20(40.0)$ & \multirow[t]{2}{*}{$>0.05$} & $21(42.0)$ & \multirow[t]{2}{*}{$>0.05$} \\
\hline Yes & 58 & $29(50.0)$ & & $24(41.3)$ & \\
\hline
\end{tabular}

${ }^{\mathrm{a}} P<0.05$ and ${ }^{\mathrm{b}} P<0.01$ when compared to adenomatous canceration; ${ }^{\mathrm{c}} P<0.05$ when compared to well-differentiation adenocarcinoma.

cases of pericancerous tissues, and 35 cases of chronic cholecystitis. Positive CDX2 staining was seen in the nuclei of carcinoma cells, as shown in Fig. 1. Hep staining was observed in the cytoplasm and/or cell membrane with brown-yellow granules as shown in Fig. 2 (a and b). For comparison, a typical Hep positive staining of hepatocytes from a liver section was shown in Fig. 2c. Overall, of the 108 cases of gallbladder adenocarcinomas, expression of CDX2 and Hep was detected in 49 (45.4\%) and 45 (41.7\%) cases, respectively. In the 46 cases of pericancerous tissues, CDX2 and Hep were positive in $13(28.3 \%)$ and 11 $(23.9 \%)$ cases, respectively. However, in the 35 cases of chronic cholecystitis, CDX2 and Hep stains were positive in $5(14.3 \%)$ and $2(5.7 \%)$ cases. Statistically, the positive rates of CDX2 and Hep in gallbladder adenocarcinoma were significantly higher than those in peritumoral tissues $\left(\chi^{2}=3.93, P<0.05\right.$ and $\chi^{2}=4.39, P<0.05$ respectively) and chronic cholecystitis $\left(\chi^{2}=10.87, P<0.01\right.$ and $\chi^{2}=15.49$, $P<0.01$ respectively). In addition, moderate to severe atypical hyperplasia were observed in all CDX2 and Heppositive cases of peritumoral tissues and chronic cholecystitis epitheliums. Some of the benign lesions and gallbladder adenocarcinoma showed morphological evidences of intesti- nal metaplasia, such as existence of goblet cells, secretion of mucus, and high columnar cancer cells.

Expressions of CDX2 and Hep and Their Correlation With Clinicopathologic Parameters of Gallbladder Adenocarcinoma

We further analyzed whether expression of CDX2 and Hep was correlated with the clinical and pathological features of gallbladder adenocarcinoma. As shown in Table 1, the frequencies of CDX2 and Hep expression in the cases with adenomatous canceration, maximal diameter of mass $<2 \mathrm{~cm}$, and no-metastasis of lymph node were significantly higher than those in the ones characterized by poorly-differentiated adenocarcinoma, maximal diameter of mass $\geq 2 \mathrm{~cm}$, and metastasis of lymph node $(P<0.05$ or $P<0.01)$. The positive rate of CDX2 in cases with no-invasive regional tissues was significantly higher than that in cases with invasive regional tissues $(P<0.01) .37$ cases were Hep-positive in the 49 CDX2-positive cases, and highly consistency in CDX2 and Hep expression was found in gallbladder adenocarcinoma $\left(\chi^{2}=42.27, P<0.01\right)$. 

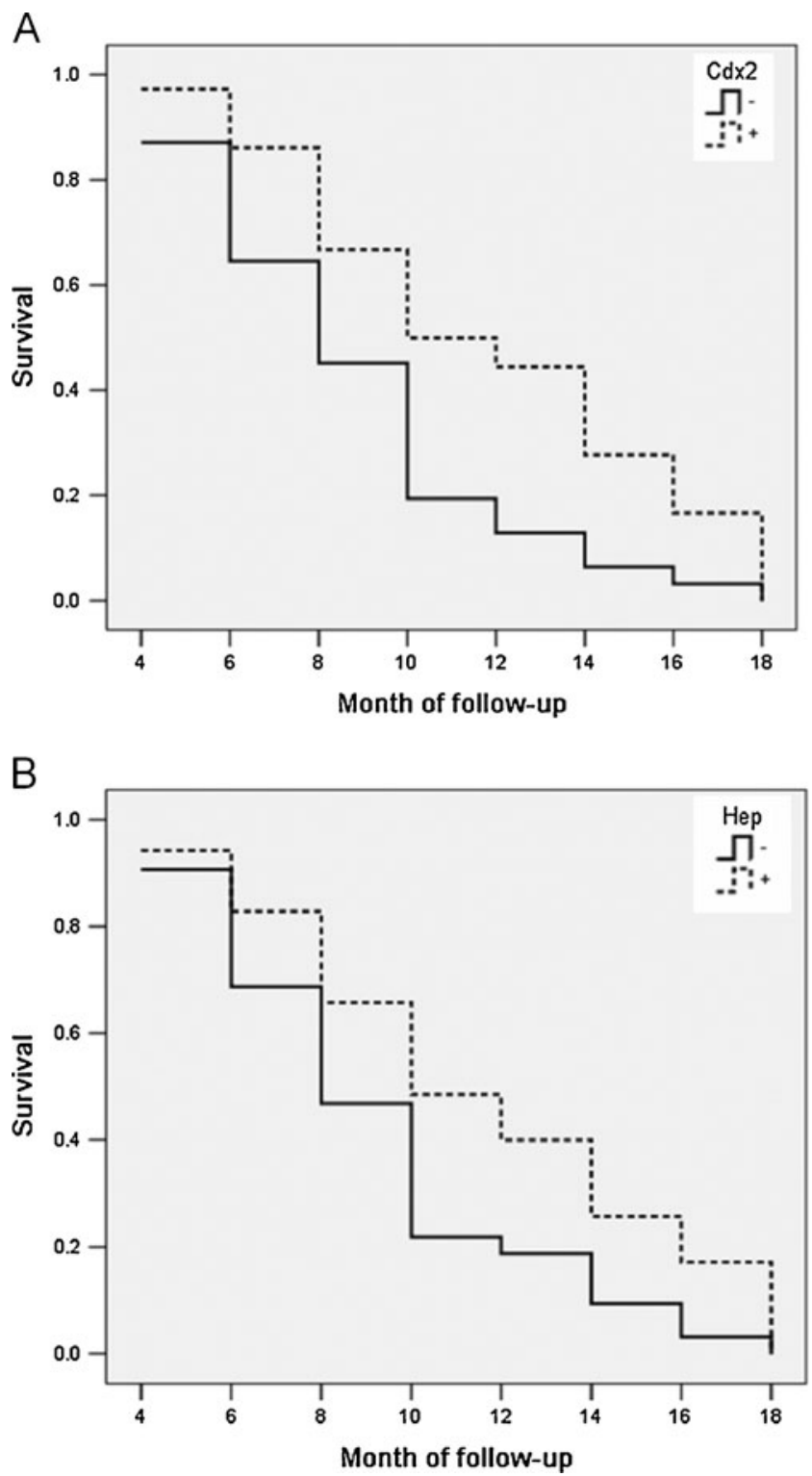

Fig. 3 Kaplan-Meier plots for overall survival in 67 patients with gallbladder adenocarcinoma surgery in relation to CDX2 and Hep expression. a Positive or increased CDX2 expression was associated with increased over survival $(P=0.003, \log$ rank test). b Hep expression was also related to increased overall survival $(P=0.02$, $\log$ rank test)

\section{Correlation of CDX2 and Hep Expression with Disease} Specific Overall Survival

After surgical resection, only 67 out of 108 patients were able to be followed up via phone or mail surveys. Of the 67 cases with follow-up, 20 cases had over 1 year survival, and 47 died within 1 year after surgery, with a mean survival time of $9.6 \pm 5.2$ months. 36 of the $67(53.7 \%)$ patients had positive staining of CDX2 and $35(52.2 \%)$ had Hep positive staining. The relevance of positive CDX2 and Hep expression to patients' survival was examined by univariate
Kaplan-Meier survival analysis. Overall survival was positive association with increased expression frequencies of CDX2 ( $P=0.003)$ (Fig. 3a) and Hep $(P=0.020)$ (Fig. 3b). The relevance of overall survival and other clinicopathological characteristics were also assessed by univariate analysis which showed that the overall survival was associated with tumor pathological type $(P=0.031)$, tumor diameter $(P=0.003)$, lymph node metastasis $(P=0.005)$ and surrounding tissue invasion $(P=0.002)$. All factors that showed significant association in the univariate KaplanMeier analysis were subsequently subject to multivariate Cox regression survival analysis, which indicated that lymph node metstasis and surrounding tissue invasion were the most significant predictors of short overall survival followed by CDX2 and Hep expression (Table 2).

\section{Discussion}

Under normal conditions, CDX2 expression is intestinespecific and plays an important role in the regulation of cell proliferation and differentiation in the embryo and gut. Recent studies demonstrated that ectopic CDX2 expression has been observed in intestinal metaplasia and carcinoma of the stomach, it might be related to gastric mucosa transformation from gastric to intestinal phenotype, and gastric carcinogenesis $[9,10,13]$. CDX2 expression was not detected in normal gallbladder mucosa [17]. We reported here that ectopic CDX2 expression was detected in $49 / 108(45.4 \%)$ of gallbladder adenocarcinoma. In the 108 cases of gallbladder carcinoma, there was no differential frequency of CDX2 expression in different sex groups, age, and with or without gallstones. However, we observed there was a trend of increasing expression frequency of $\mathrm{CDX} 2$ in benign lesions and CDX2 was strongly relevant to grades of tumor differentiation.

CDX2 expression has been considered as one of the good prognostic markers in patients with gastric carcinoma $[13,26,27]$, pancreatic tumor [28], and carcinoma of the ampulla of Vater [29]. Few studies have examined CDX2 expression in gallbladder cancer specimens. Wu et al. reported that CDX2 expression was detected in 25 (36.8\%) of 68 gallbladder carcinomas, and was closely related to histological differenation grades, i.e., in the tubular type gallbladder carcinomas, significantly higher expression in well-differentiated type than the moderately and poorly differentiated types, indicating that ectopic expression of CDX2 is an early event in gallbladder carcinomas. However, whether CDX2 can be a prognostic factor remained unclear in their study due to limited case number obtained [17]. Jinawath et al. have found that CDX2 expression was observed in $22(37.3 \%)$ of 59 cholangiocarcinoma cases, and correlated with MUC2 expression. 
Table 2 Multivariate cox regression analysis of overall survival in 67 patients with surgical resection of gallbladder carcinoma

\begin{tabular}{|c|c|c|c|c|c|c|c|}
\hline \multirow[t]{2}{*}{ Group } & \multirow[t]{2}{*}{ Category } & \multirow[t]{2}{*}{ B } & \multirow{2}{*}{$\begin{array}{l}\text { SE } \\
\text { (B) }\end{array}$} & \multirow{2}{*}{$\begin{array}{l}\text { Exp } \\
\text { (B) }\end{array}$} & \multirow[t]{2}{*}{$\mathrm{P}$} & \multicolumn{2}{|c|}{$95 \%$ CI for $\operatorname{Exp}(B)$} \\
\hline & & & & & & Inferior & Superior \\
\hline Pathology type & $\begin{array}{l}\text { Adenoma canceration/well-/moderately-/ } \\
\text { poorly-differentiatied/mucous carcinoma }\end{array}$ & 0.508 & 0.378 & 1.66 & 0.253 & 0.79 & 3.49 \\
\hline Tumor Diameter & $<2.0 \mathrm{~cm} / \geq 2.0 \mathrm{~cm}$ & 0.723 & 0.301 & 2.06 & 0.04 & 1.14 & 3.72 \\
\hline $\begin{array}{l}\text { Lymphonode } \\
\text { metastasis }\end{array}$ & No/Yes & 0.958 & 0.302 & 2.61 & 0.013 & 1.44 & 4.71 \\
\hline $\begin{array}{l}\text { Surrounding tissue } \\
\text { invasion }\end{array}$ & No/Yes & 0.911 & 0.308 & 2.49 & 0.027 & 1.36 & 4.55 \\
\hline $\mathrm{CDX} 2$ & $-/+$ & -0.852 & 0.350 & 0.43 & 0.014 & 0.21 & 0.84 \\
\hline Hep Par 1 & $-/+$ & -0.185 & 0.056 & 0.82 & 0.026 & 0.72 & 0.94 \\
\hline
\end{tabular}

The expression of CDX2 was much higher in papillary-type than tubular-type cholangiocarcinoma [14]. Additionally, Hong et al. have shown that CDX2 was detected in 72 $(37.3 \%)$ out of 193 extraHepatic bile duct carcinomas and correlated with histologic subtypes, and more frequently in tumors with papillary growth and no vascular invasion. They further concluded that Patients with CDX2+/MUC2+ tumors had significantly better overall survival in univariate but not multivariate analysis than patients with other tumors [30]. Chang et al. demonstrated that 27/164 (16.46\%) patients with biliary tract carcinoma expressed CDX2, and the frequency of CDX2 expression was much higher in gallbladder carcinoma $(12 / 41,29.3 \%)$ and extrahepatic cholangiocarcinoma $(22.45 \%)$ than in the intrahepatic cholangiocarcinoma [16]. By univariate and multivariate analyses, the expression of CDX2 was associated with a significant survival advantage; CDX2 and tumor stage were independent prognostic factor in patients with biliary tract carcinomas [16]. In our study, CDX2 expression was associated with tumor differentiation stages, it was found much higher in benign lesions, i.e. adenomous canceration and well-differentiated types, than the poor-differentiated type. Additionally, CDX2 expression was associated with better over survival patients with gallbladder carcinomas, and multivariate Cox regression analysis showed that CDX2 was an independent predictor of survival in addition to lymph node metstasis and surrounding tissue invasion after resection of gallbladder tumor.

Hep is a component of the membrane of hepaticcellular mitochondrial. The positive rates of Hep in normal hepatic cells and cells of chronic hepatic diseases were $100 \%$, and the positive rate of Hep in hepatocellular carcinoma was above $90 \%$, especially that in well-differentiated Hepatocellular carcinoma was nearly $100 \%$, so Hep was regarded as an important marker for diagnosis of hepatocellular carcinoma and differential diagnosis between hepatocellular carcinoma and metastatic carcinoma of liver, and positive cases had better prognosis than negative cases [8]. Recent studies have found that Hep had high expression in many other malignant tumors, such as intestinal gastric cancer and esophagus adenocarcinoma with intestinal metaplasia, and Hep was regarded as a specific marker reflecting intestinal metaplasia of gastric mucosa; malignant tumors with higher Hep expression had better prognosis, and Hep was regarded as a objective biological marker reflecting the prognosis of malignant tumors [13, 18, 24, 31-33]. To our knowledge, quite few literatures have reported the expression of Hep in gallbladder carcinoma, in nomoral condition there is no Hep expression detected in gallbladder tissue. In gallbladder adenocarcinomas, the Hep expression rate from 0 to $100 \%$ have been reported [20-23]. When Leong et al. used Hep par 1 to distinct hepatocellular carcinoma from cholangiocarcinoma, combined tumors and metastatic carcinoma, they did not detect Hep expression in 2 cases of metastic gallbladder adenocarcinomas [20]. Neither did Mosnier et al. in their study with 6 cases of gallbladder adenocarcinomas [21]. However, Maitra et al. and Lugli et al. reported positive rate $100 \%$ and $26 \%$ of Hep expression in gallbladder adenocarcinomas, respectively [22, 23]. Our results showed that Hep expression was detected in 45 (41.7\%) out of 108 gallbladder adenocarnoma cases, which is close to Lugli et al. reported 26\% [23]. We found out the expression rate of Hep in gallbladder adenocarcinoma was significantly higher than those in peritumoral tissues (23.9\%) and chronic cholecystitis (5.7\%). Hep expression was positively associated with well-differentiated, small size and no metastasis tumors. Most of positive cases showed intestinal metaplasia., suggesting Hep expression is associated with intestineal metaplasia. The gallbladder lesions with Hep expression was significantly correlated to early stage, small size, well differentiation, and no-metastasis.

Highly consistency in CDX2 and Hep expression was found in gallbladder adenocarcinoma. Unitivariate KaplanMeier analysis and Multivariate Cox regression analysis showed that increased expression of Hep also was an 
independent better-prognostic factor. The result suggested that some gallbladder adenocarcinoma expressed high level of Hep, which might be related to intestinal metaplasia of gallbladder epithelium, and Hep might be one of the important biological markers reflecting the prognosis of gallbladder adenocarcinoma. In addition, highly consistency in CDX2 and Hep expression might because that they were both biological markers of intestinal metaplasia.

In summary, our findings indicate that expression of CDX2 or Hep expression influenced the overall survival in patients with gallbladder carcinoma. Both CDX2 and Hep expression were independent markers of clinical outcome in addition to lymph node metstasis and surrounding tissue invasion in gallbladder adenocarcinoma patients, and might be useful to predict the prognosis for gallbladder carcinoma.

Open Access This article is distributed under the terms of the Creative Commons Attribution Noncommercial License which permits any noncommercial use, distribution, and reproduction in any medium, provided the original author(s) and source are credited.

\section{References}

1. Jones RS (1990) Carcinoma of the gallbladder. Surg Clin North Am 70:1419-1428

2. Carriaga MT, Henson DE (1995) Liver, gallbladder, extraHepatic bile ducts, and pancreas. Cancer 75:171-190

3. Suh E, Chen L, Taylor J, Traber PG (1994) A homeodomain protein related to caudal regulates intestine-specific gene transcription. Mol Cell Biol 14:7340-7351

4. Silberg DG, Swain GP, Suh ER, Traber PG (2000) Cdx1 and cdx2 expression during intestinal development. Gastroenterology 119:961-971

5. Phillips RW, Frierson HF Jr, Moskaluk CA (2003) Cdx2 as a marker of epithelial intestinal differentiation in the esophagus. Am J Surg Pathol 27:1442-1447

6. Eda A, Osawa H, Satoh K et al (2003) Aberrant expression of CDX2 in Barrett's epithelium and inflammatory esophageal mucosa. J Gastroenterol 38:14-22

7. Bai YQ, Yamamoto H, Akiyama Y et al (2002) Ectopic expression of homeodomain protein $\mathrm{CDX} 2$ in intestinal metaplasia and carcinomas of the stomach. Cancer Lett 176:47-55

8. Mutoh H, Hakamata Y, Sato K et al (2002) Conversion of gastric mucosa to intestinal metaplasia in $\mathrm{Cdx} 2$-expressing transgenic mice. Biochem Biophys Res Commun 294:470-479

9. Kim HS, Lee JS, Freund JN et al (2006) CDX-2 homeobox gene expression in human gastric carcinoma and precursor lesions. J Gastroenterol Hepatol 21:438-442

10. Vallbohmer D, DeMeester SR, Peters JH et al (2006) Cdx-2 expression in squamous and metaplastic columnar epithelia of the esophagus. Dis Esophagus 19:260-266

11. Nonaka D, Kusamura S, Baratti D, Casali P, Younan R, Deraco M (2006) CDX-2 expression in pseudomyxoma peritonei: a clinicopathological study of 42 cases. Histopathology 49:381387
12. Ishikawa A, Sasaki M, Ohira S et al (2004) Aberrant expression of CDX2 is closely related to the intestinal metaplasia and MUC2 expression in intraductal papillary neoplasm of the liver in Hepatolithiasis. Lab Investigation Journal Technical Methods Pathology 84:629-638

13. Fan Z, Li J, Dong B, Huang X (2005) Expression of Cdx2 and Hepatocyte antigen in gastric carcinoma: correlation with histologic type and implications for prognosis. Clin Cancer Res 11:6162-6170

14. Jinawath A, Akiyama Y, Yuasa Y, Pairojkul C (2006) Expression of phosphorylated ERK1/2 and homeodomain protein CDX2 in cholangiocarcinoma. J Cancer Res Clin Oncol 132:805-810

15. Sakamoto H, Mutoh H, Ido K, Satoh K, Hayakawa H, Sugano K (2007) A close relationship between intestinal metaplasia and $\mathrm{Cdx} 2$ expression in human gallbladders with cholelithiasis. Hum Pathol 38:66-71

16. Chang YT, Hsu C, Jeng YM, Chang MC, Wei SC, Wong JM (2007) Expression of the caudal-type homeodomain transcription factor $\mathrm{CDX} 2$ is related to clinical outcome in biliary tract carcinoma. J Gastroenterol Hepatol 22:389-394

17. Wu XS, Akiyama Y, Igari $\mathrm{T}$ et al (2005) Expression of homeodomain protein CDX2 in gallbladder carcinomas. J Cancer Res Clin Oncol 131:271-278

18. Chu PG, Ishizawa S, Wu E, Weiss LM (2002) Hepatocyte antigen as a marker of Hepatocellular carcinoma: an immunohistochemical comparison to carcinoembryonic antigen, CD10, and alphafetoprotein. Am J Surg Pathol 26:978-988

19. Kakar S, Gown AM, Goodman ZD, Ferrell LD (2007) Best practices in diagnostic immunohistochemistry: Hepatocellular carcinoma versus metastatic neoplasms. Arch Pathol Lab Med 131:1648-1654

20. Leong AS, Sormunen RT, Tsui WM, Liew CT (1998) Hep Par 1 and selected antibodies in the immunohistological distinction of Hepatocellular carcinoma from cholangiocarcinoma, combined tumours and metastatic carcinoma. Histopathology 33:318-324

21. Mosnier JF, Kandel C, Cazals-Hatem D et al (2009) N-cadherin serves as diagnostic biomarker in intraHepatic and perihilar cholangiocarcinomas. Mod Pathol 22:182-190

22. Maitra A, Murakata LA, Albores-Saavedra J (2001) Immunoreactivity for Hepatocyte paraffin 1 antibody in Hepatoid adenocarcinomas of the gastrointestinal tract. Am J Clin Pathol 115:689-694

23. Lugli A, Tornillo L, Mirlacher M, Bundi M, Sauter G, Terracciano LM (2004) Hepatocyte paraffin 1 expression in human normal and neoplastic tissues: tissue microarray analysis on 3,940 tissue samples. Am J Clin Pathol 122:721-727

24. Fan Z, van de Rijn M, Montgomery K, Rouse RV (2003) Hep par 1 antibody stain for the differential diagnosis of Hepatocellular carcinoma: 676 tumors tested using tissue microarrays and conventional tissue sections. Mod Pathol 16:137-144

25. Mac MT, Chung F, Lin F, Hui P, Balzer BL, Wang HL (2009) Expression of Hepatocyte antigen in small intestinal epithelium and adenocarcinoma. Am J Clin Pathol 132:80-85

26. Mizoshita T, Tsukamoto T, Nakanishi H et al (2003) Expression of $\mathrm{Cdx} 2$ and the phenotype of advanced gastric cancers: relationship with prognosis. J Cancer Res Clin Oncol 129:727734

27. Seno H, Oshima M, Taniguchi MA et al (2002) CDX2 expression in the stomach with intestinal metaplasia and intestinal-type cancer: prognostic implications. Int $\mathrm{J}$ Oncol 21:769-774

28. Matsumoto K, Mizoshita T, Tsukamoto T et al (2004) Cdx2 expression in pancreatic tumors: relationship with prognosis of invasive ductal carcinomas. Oncol Rep 12:1239-1243 
29. Hansel DE, Maitra A, Lin JW et al (2005) Expression of the caudal-type homeodomain transcription factors CDX $1 / 2$ and outcome in carcinomas of the ampulla of Vater. J Clin Oncol 23:1811-1818

30. Hong SM, Cho H, Moskaluk CA, Frierson HF Jr, Yu E, Ro JY (2005) CDX2 and MUC2 protein expression in extraHepatic bile duct carcinoma. Am J Clin Pathol 124:361-370

31. Kakar S, Muir T, Murphy LM, Lloyd RV, Burgart LJ (2003) Immunoreactivity of Hep Par 1 in Hepatic and extraHepatic tumors and its correlation with albumin in situ hybridization in Hepatocellular carcinoma. Am J Clin Pathol 119:361-366

32. Ramos-Vara JA, Miller MA (2002) Immunohistochemical characterization of canine intestinal epithelial and mesenchymal tumours with a monoclonal antibody to Hepatocyte paraffin 1 (Hep Par 1). Histochem J 34:397-401

33. Hishinuma M, Ohashi KI, Yamauchi N et al (2006) Hepatocellular oncofetal protein, glypican 3 is a sensitive marker for alphafetoprotein-producing gastric carcinoma. Histopathology 49:479-486 